\title{
Effect of Net-Fluence on waveguide formation in ultrafast laser inscribed chalcogenide glass
}

\author{
Tamilarasan Sabapathy $^{1}$, Gayathri Sivakumar ${ }^{1}$, Arunbabu Ayiriveetil ${ }^{1}$, Ajoy K Kar ${ }^{2}$ \\ and Sundarrajan Asokan ${ }^{1,3}$ \\ ${ }^{1}$ Department of Instrumentation and Applied Physics, Indian Institute of Science, Bangalore, INDIA- 560012 \\ ${ }^{2}$ School of Engineering and Physical Sciences, Heriot-Watt University, Edinburgh EH14 4AS, UK \\ ${ }^{3}$ Applied photonics initiative, Indian Institute of Science, Bangalore, INDIA - 560012 \\ thamizhaiap@gmail.com
}

\begin{abstract}
Waveguides were fabricated on GeGaSEr chalcogenide glass using ultrafast laser inscription method. The thermal diffusion model is discussed for understanding the light matter interaction and shown the effect of net-fluence in waveguide formation on chalcogenide glass.
\end{abstract}

(C) 2012 Optical Society of America

OCIS codes: $230.3120,140.3390$

\section{Introduction}

Chalcogenide glasses are attractive because of its transparency in mid-IR region, photo-sensitivity [1], high third order non-linearity, chemically and thermally stable, easy to dope with rare earth ions due to the high solubility and low phonon energy [2,3]. Direct laser writing method employs multi-photon absorption by focusing its sub bandgap energy [4] into the material to produce high energy to cause structural changes in the material for fabricating devices like waveguides, lasers and amplifiers on bulk transparent dielectric material. A major advantage of fabricating waveguides using femtosecond micromachining is the ability to create 3-D structures inside the transparent materials [5].

Bulk GeGaSEr glass prepared by vacuum sealed conventional melt quenching technique. The amorphous nature of the sample was confirmed by X-ray diffraction. In this work, controlling the waveguide cross section by solving the heat diffusion model by finite difference method is discussed. From this model, we compared the theoretical values with the experimental results for waveguide cross-section.

\section{Waveguide fabrication and Charecterization}

We fabricated waveguides on GeGaSEr chalcogenide glass using Yb-doped femtosecond fiber laser. A circularly polarized sub-picosecond pulse laser of $1047 \mathrm{~nm}$ wavelength, $350 \mathrm{fs}$ pulse duration at repetition rate of $100 \mathrm{kHz}$ were set for inscription. Waveguides were fabricated using high intensity laser pulses. The laser pulses were focussed using asperic lens having $0.68 \mathrm{NA}$ at a distance of $100 \mu \mathrm{m}$ below the surface of the GeGaSEr glass sample. Each waveguide is separated by $50 \mu \mathrm{m}$ to avoid the overlapping or stress influence on the subsequent waveguide. The various range of powers from $94 \mathrm{~mW}$ to $30 \mathrm{~mW}$ by $85 \%$ step down and different translation speeds of $18 \mathrm{~mm} / \mathrm{s}, 10 \mathrm{~mm} / \mathrm{s}, 6 \mathrm{~mm} / \mathrm{s}$ and $4 \mathrm{~mm} / \mathrm{s} 1 \mathrm{~mm} / \mathrm{s}$ and $0.1 \mathrm{~mm} / \mathrm{s}$ were used to inscribe the waveguide.

The near field image of the waveguide was captured at a wavelength of $1550 \mathrm{~nm}$, using IR camera with 0.65 NA objective lens. The optically induced structure was captured by an optical microscope at 50x magnification. The corresponding images are provided in Fig. 1.

\section{Modeling}

The temperature distribution during waveguide formation was calculated with a finite-difference thermal diffusion model governed by the thermal diffusion partial differential equation with spherical symmetry

$$
\frac{\partial}{\partial r}\left(r^{2} \frac{\partial T}{\partial r}\right)=\frac{r^{2}}{D}\left(\frac{\partial T}{\partial t}\right)
$$



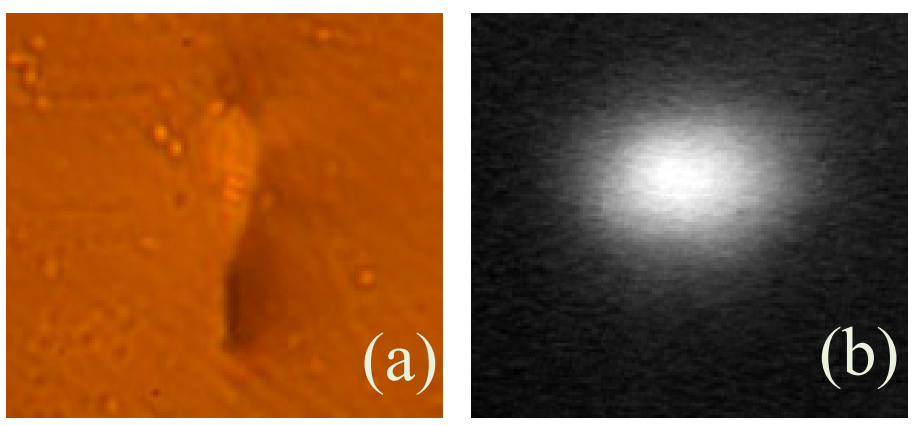

Fig. 1. (a) Optical microscope image of single mode waveguide and (b) its corresponding near field image at $1550 \mathrm{~nm}$

The laser dissipation was treated as a spherical Gaussian distribution with energy volume density $E(r)=$ $E_{0} \exp \left(-r^{2} / w_{0}\right)$ with beam waist $\mathrm{w}_{0}$ set to the $1 / e^{2}$ radius of the focused laser beam waist. The value of $E_{0}$ depends on the absorbed laser pulse energy, which was inferred from measurement of the transmitted laser power. The values of $\mathrm{E}_{0}$ were varied by changing the the laser power, scan speed, and repetition rate. The temperature profile was augmented by an instantaneous temperature rise $T(r)=E(r) / C_{p} \rho$, each time a new laser pulse arrived. To model the effect of net fluence on waveguide diameter, specific heat capacity $\left(673 \mathrm{~J} / \mathrm{g}^{\circ} \mathrm{C}\right)$, thermal diffusivity $(0.0026 \mathrm{~cm} / \mathrm{s})$ and density $\left(2761.1 \mathrm{~kg} / \mathrm{m}^{3}\right)$ of the material were calculated using modulated DSC, a custom made photothermal diffusivity instrument and Metler Toledo density Kit respectively.

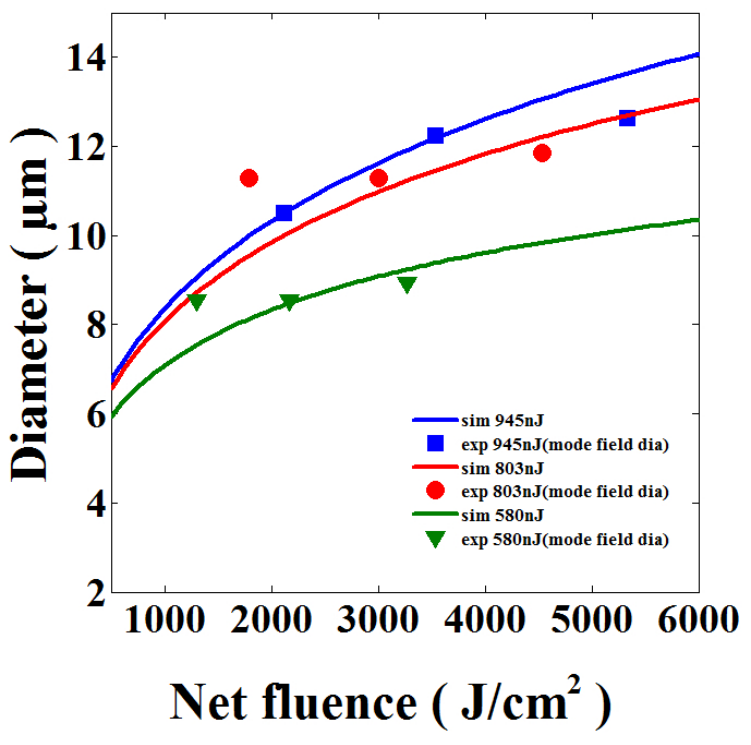

Fig. 2. The relation between Waveguide diameter and net fluence for experimentally observed and theoretically calculated values

\section{Discussion}

The waveguide near field image at $1550 \mathrm{~nm}$ wavelength was captured using IR camera with 0.65 NA objective lens and the optically induced structure are captured with the help of optical microscope with 50x magnification (Fig.1). We calculated the waveguide diameter by comparing pixel values of the near field images of the standard telecommunication 
fiber and the waveguide.

The finite-difference heating model is used to find the waveguide spatial formation [6]. To compare this model with the experimental results, net fluence (NF) is calculated for each energy and translation speed. The net fluence is calculated using the following relation,

$$
N F=\frac{2 R}{\pi \omega_{0}} \frac{E_{p}}{v}
$$

where $\omega_{0}$ is the beam waist, $\mathrm{R}$ is repetition rate and $v$ is the translation speed and $\mathrm{E}_{p}$ is the incident laser pulse energy.

Fig. 2 shows the relation between the net-fluence and the diameter of the waveguide from solving the thermal diffusion equation [6,7]. We have taken three energy with four different translation speed for the modeling. The model best fits the experimental data with $70 \%, 80 \%$ and $90 \%$ absorption for 580, 803 and $945 \mathrm{~nJ}$ of incident pulse energies respectively. For the incident pulse energies $580 \mathrm{~nJ}, 803 \mathrm{~nJ}$, and $945 \mathrm{~nJ}$ the corresponding absorbed laser energies were $406 \mathrm{~nJ}, 674 \mathrm{~nJ}$, and $850 \mathrm{~nJ}$ repsctively. This suggest that with increase in incident pulse energy the absorption also increases, which further leads to an higher initial temperature. As expected, the transverse waveguide diameter increases with increasing net exposure, through decreasing scan speed. With increase in absorbed energy, larger waveguide diameter were observed (Fig. 1a). This could be attributed to the strong heat diffusion effects owing to an increases photoionization rate. There is a fine agreement between theoretical and experimental values of waveguide diameter. The theoretical model data goes well with experimental data within $7 \%$ error at $945 \mathrm{~nJ}$ pulse energy. At 803 $\mathrm{nJ}$ and $580 \mathrm{~nJ}$ pulse energy the estimated values vary about $18 \%$ and $13 \%$ respectively from the experimental results.

\section{Conclusion}

We presented the waveguide formation in GeGaSEr chalcogenide glass due to the thermal diffusion using ultrafast laser inscription method at low repetition rate. The relation between the net fluence and waveguide cross section diameter was verified using these experimentally measured physical properties. The theoretically predicted values are in agreement with the experimental values.

\section{References}

1. C. D Lezal, "Chalcogenide glasses - survey and progress," J. Optoelectron. Adv. Mater. 5, 23-24 (2003).

2. A. Zakery, and S. R. Elliott,'Optical properties and applications of chalcogenide glasses: a review," J. NonCryst. Solids 330, 1-12 (2003).

3. A. Zakery and S. R. Elliott, Optical Nonlinearities in Chalcogenide Glasses and their Applications (Springer Series in Optical Sciences, 2007).

4. R. R. Gattass and E. Mazur,"Femtosecond laser micromachining in transparent materials," Nat. Photonics 2, 219-225 (2008).

5. K. M. Davis, K. Miura, N. Sugimoto, and K. Hirao,’Writing waveguides in glass with a femtosecond laser," Opt. Lett. 21, 1729 (1996).

6. Shane M. Eaton, Haibin Zhang, Mi Li Ng, Jianzhao Li, Wei-Jen Chen, Stephen Ho, and Peter R. Herman,"Transition from thermal diffusion to heat accumulation in high repetition rate femtosecond laser writing of buried optical waveguides," Opt. Express 16, 9443-9458 (2008).

7. C. B. Schaffer, J. F. Garcia, and E. Mazur,'Bulk heating of transparent materials using a high-repetition-rate femtosecond laser,' Appl. Phys. A 76, 351-354 (2003). 